\title{
Applying ZigBee Technology to the Design of Marine Engine Room Monitoring Systems
}

\author{
Mingyu $\operatorname{TIAN}^{1, \mathrm{a}}$, Hao $\mathrm{CAl}^{2, \mathrm{~b}}$ and Hongbing $\mathrm{ZOU}^{3, \mathrm{c}^{*}}$ \\ ${ }^{1}$ School of Energy \& Power Engineering, Wuhan University of Technology, Wuhan, China \\ ${ }^{2}$ School of Computer Science \& Technology, Wuhan University of Technology, Wuhan, China \\ ${ }^{3}$ School of Navigation, Wuhan University of Technology, Wuhan, China \\ atianmingyu@whut.edu.cn, bcaihao@whut.edu.cn, 'bhzsin@163.com
}

\begin{abstract}
Keywords: ZigBee technology, marine engine room, monitoring system.
Abstract. The monitoring and alarming system of a marine engine room plays a significant role in the shipping automation. However, the performance of a monitoring system depends largely on the development and support of both hardware- and software-based technologies. Different kinds of problems exist in the traditional monitoring and alarming system of a marine engine room such as complex wiring, blind communication area, etc. In this paper, a new wireless monitoring system is designed based on ZigBee technology in order to properly and effectively conduct the temperature monitoring of major electric devices in a ship engine room. The proposed system takes the CC2430 module as the core component. In addition, the design of software design is achieved based on ZigBee Protocol. This research will provide a helpful reference for the decision-making in machinery maintenance, and facilitate the development of intelligent marine engine room in the future.
\end{abstract}

\section{Introduction}

As one of the main components of shipping automation, the monitoring and alarming system of a marine engine room is able to accurately and reliably monitor the operation condition of various kind of equipment and systems such as main propulsion systems, power generations, and auxiliary equipment within the engine room, and automatically sound the alarms once any of the equipment or systems fail to run properly [1]. In the 1960s, the process industry suffered from a low level of control automation, when conventional mechanical instruments were used for monitoring purpose. One typical representative of that time is relay-based monitoring alarm systems. The development and extensive application of computers in the 1980s facilitated the usage of advanced control systems, and make the application of microcomputer-based distributed monitoring systems in marine engine room available. At the beginning of 21 st Century, with the development of Wireless Sensor Networks (WSN), the monitoring and alarming system of a marine engine room has transformed from wired and centralized systems to wireless sensor network-based ones, so that the traditional problems such as narrow wiring space onboard ships and difficulties during construction process can be effectively solved [2].

ZigBee is an IEEE 802.15.4-based specification (which includes four additional key components: network layer, application layer, ZigBee Device Objects (ZDOs) and manufacturer-defined application objects.) for a suite of high-level communication protocols that aims at remote control and sensor applications which is suitable for operation in harsh radio environments and in isolated locations. ZigBee is a reliable and ideal proposal used in a low rate, low complexity, low cost, and low consumption wireless communication [3]. Due to the above advantages, its industrial applications can be widely seen in a variety of fields [4] including home entertainment and control, wireless sensor networks, smoke and temperature monitoring, to name just a few.

Wireless sensor networks used for monitoring purpose usually work under the condition of small amounts of data. However, a high transmission reliability is required, and other requirements include low costs and small size of the equipment used, battery-powered, and the ability to achieve complex layout, multi-monitoring points, and a wide network coverage. Therefore, the above-mentioned 
characteristics of ZigBee technology make it a superior choice in terms of its application in the WSNs. In view of that, this paper proposes a design for the marine engine room monitoring systems based on ZigBee technology [5-6].

\section{Hardware design}

The main purpose of this study is to achieve the monitoring of temperature of marine engine rooms by using the WSN composed of CC2430 module and the DS18B20 sensor. The basic components of the WSN system is a network terminal node consisting of DS18B20 temperature sensor and ZigBee module, which periodically collects temperature data as required and transmits it to a coordinator via ZigBee technology, and then to the monitor so as to monitor the temperature and achieve the alarm of abnormal temperature.
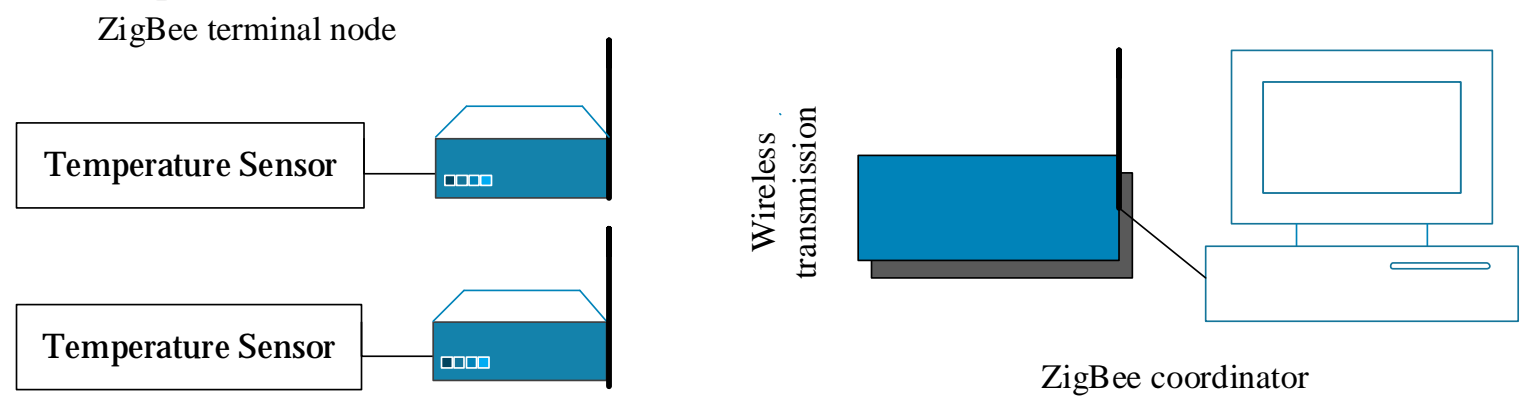

Fig. 1 Network architecture of wireless temperature sensors

The wireless temperature sensor network of the system is built upon ZigBee terminals and a ZigBee coordinator. All ZigBee terminals are connected to the coordinator, and the coordinator are connected to the PC through a serial port, as shown in Fig. 1. The terminal node of the system is composed of the CC2430 module and the temperature sensor DS18B20. CC2430 chips, their peripheral circuit, and antenna are integrated to the CC2430 module. The environmental temperature information collected by the temperature sensor will be transmitted to the memory unit of CC 2430 chips, and then sent by the wireless communication module to the coordinator. Finally, temperature information received by the coordinator will be transmitted to the computer promptly.

Design of CC2430's power module. As the power requirement of CC2430 is between 2.0 to $3.6 \mathrm{~V}$, the sensor node generally adopts the direct-flow voltage source of 3.3V. Due to the low energy consumption of the terminal node and complex environment, batteries are usually used. In this case, three AA batteries are used considering the energy consumption of the coordinator. In addition, a level conversion chip TPS7333Q is used to change the power supplies from $5 \mathrm{~V}$ and $4.5 \mathrm{~V}$ to $3.3 \mathrm{~V}$. No input capacitor is needed, as a $10 \mathrm{uF}$ solid tantalum capacitor between the output of the regulator and the ground is enough to maintain the stability of chip TPS7333Q. A ceramic bypass capacitors with $0.047 \mathrm{PF} \sim 0.1 \mathrm{uF}$ is also coupled to improve load transient response and noise suppression. A schematic diagram of the power module is shown in Fig. 2.

Design of wireless temperature sensor network terminal. A waterproof digital temperature sensor DS18B20 is used to detect environment temperature, and the collected information is transferred to the computer terminal via the CC2430 module. The sensor DS18B20 is connected to the CC2430 module through circuit, making up a wireless temperature sensor network terminal node. The data line of DS18B20 is connected to P07 port of CC2430 for power supply. The connection diagram between sensor DS18B20 and CC2430 module is shown in Fig. 3. 


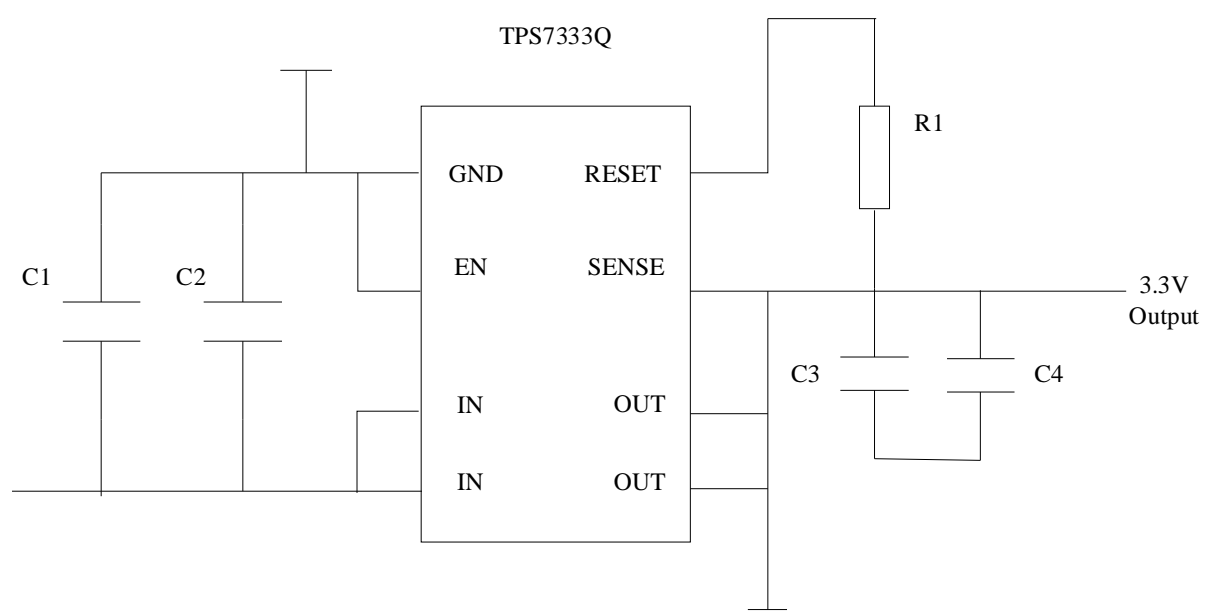

Fig. 2 A schematic diagram of the power module

In terms of the usage of DS18B20, there are strict timing requirements for data reading and writing due to the fact that both the processes occur on a single $\mathrm{I} / \mathrm{O}$ line. The strict communication protocol is provided to ensure the correctness and integrity of data transmission of it. The agreement defines the initialization sequence, read timing, and write timing. These timings have set the host as a master device and the single-bus device as the slave one. The transmission of data and command begins only when the host starts the write sequence. If the single-bus device is required to send data back, the host needs to start the read sequence to complete the data reception after executing the write command. Both the transmission of data and command is little-endian.

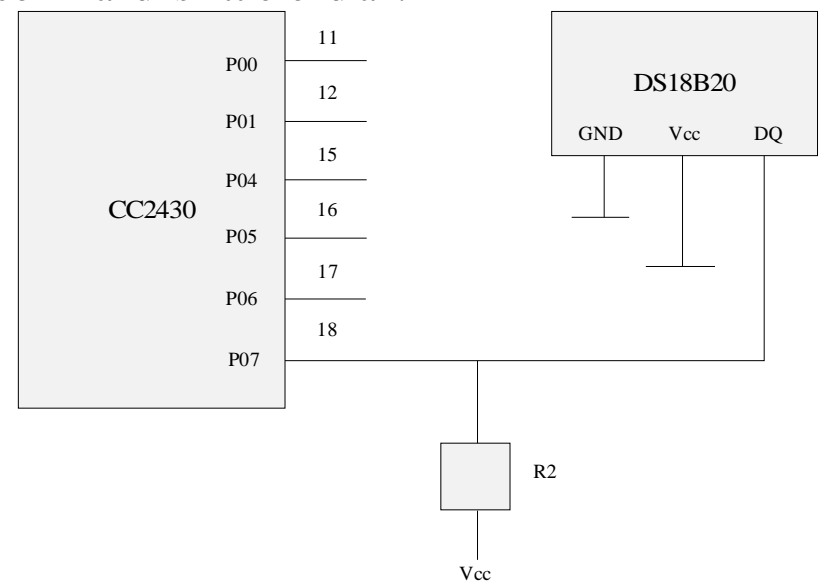

Fig. 3 A circuit diagram of connection between DS18B20 and CC2430

Computer interface circuits. Level matching is a must for CC2430 in order to communicate with the computer through the serial. The CC2430 uses the Transistor-Transistor Logic (TTL) level standard, while computer serial ports apply the RS-232 level standard. The different level range between the two devices calls for a level conversion circuit. In this study, the chip MAX3232 is used to achieve the level conversion.

\section{Software design}

Design of wireless temperature sensor network software. The objective of this paper is to monitor the temperature of a certain area in the marine engine room. The process of temperature monitoring is shown in Fig. 4. The sensor nodes of DS18B20 and CC2430 are tested every half minute, and the information is transmitted to the coordinator through the ZigBee wireless transmission protocol. After the coordinator receiving the temperature data, it is transmitted to the computer through the serial line. All temperature measurement data will be recorded on the computer, and the computer client can monitor the temperature data changes in real time. 
Terminal node

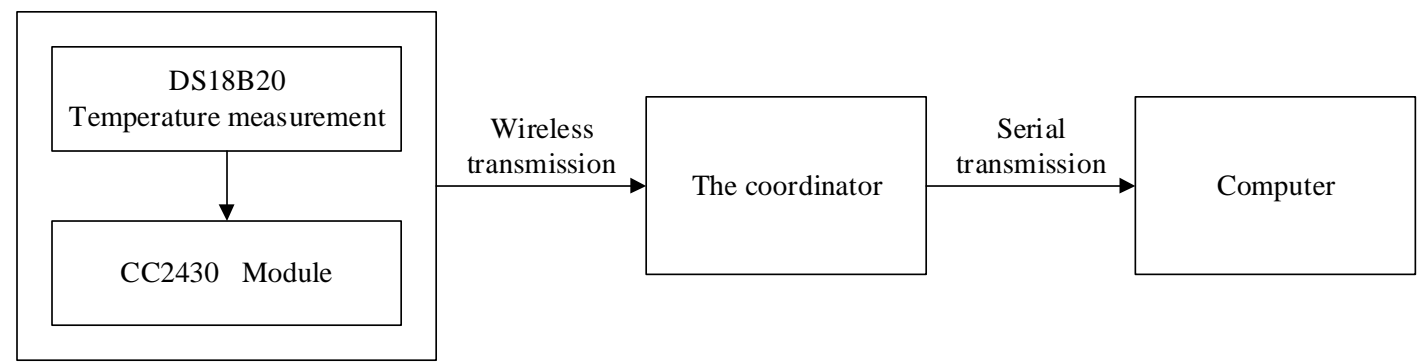

Fig. 4 Monitor temperature information and transfer flow chart

The main steps of the software are as follows: 1) Initialize the system; 2) Initialize radiofrequency (RF) module; 3) Enable sensor terminal node and the coordinator; 4) Compose the sensor network and start the temperature measurement program; 5) Transfer the temperature measurement data to the computer.

According to the hardware circuit design, the software adopts the modular structure programming method. The software module includes UART serial port module, initialization of radio frequency chip, temperature data detection, data receiving and sending of radio frequency chip, and temperature alarm threshold settings. The coordinator uses the CC2430 module to supply power with a stable DC power supply. When the coordinator is enabled, it starts to scan channel and establish a logical link. As soon as a terminal node applies to join the network, a wireless communication network can be established to transmit data, and connect to the computer through the RS232 serial port. The specific software flow is shown in Fig. 5.

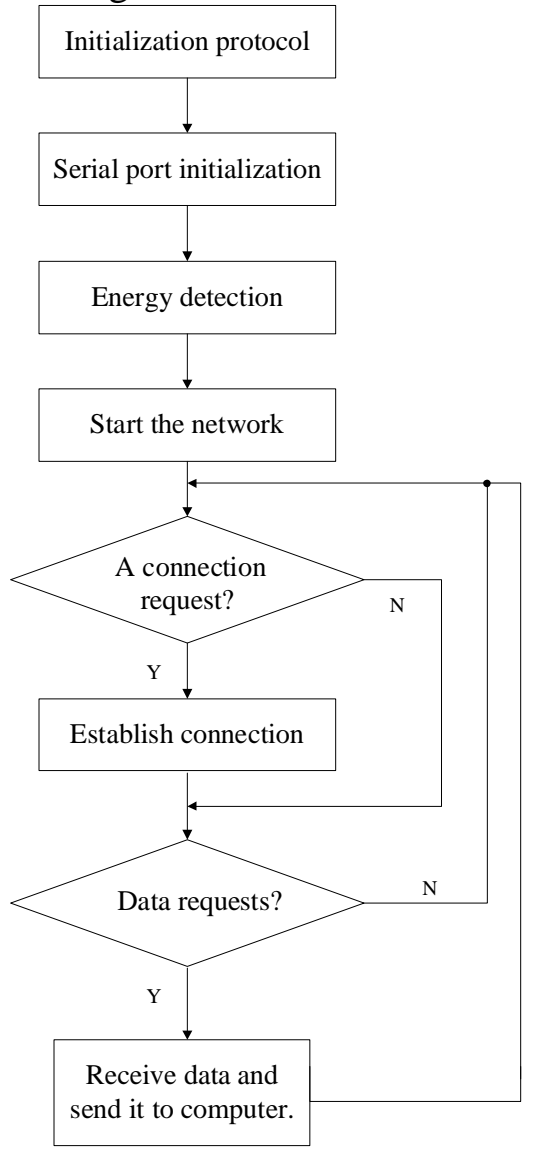

Fig. 5 The coordinator software process

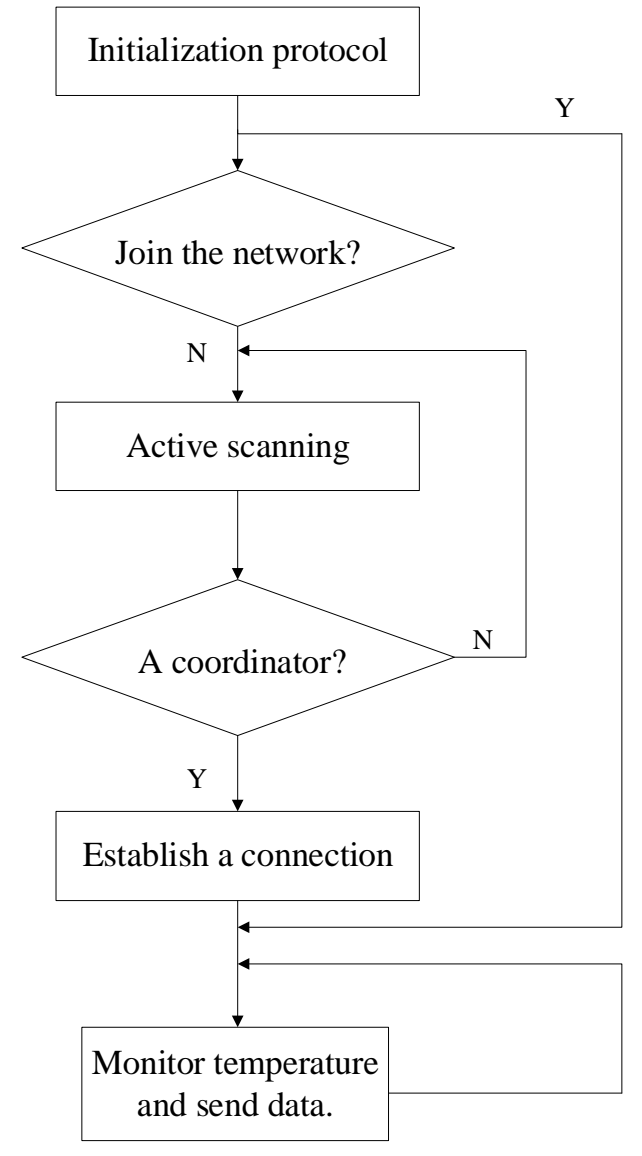

Fig. 6 Flow chart of terminal node software

The wireless temperature monitoring network terminal node is composed of CC2430 module and DS18B20, which is powered by batteries. When the terminal node is electrically reset, it starts to search for the coordinator on the specified channel and issue a connection request. If the connection is 
successful, the terminal node will get a 16-bit short address and communicate using the address. In order to save energy, the terminal node should be put into energy saving status during non-work condition. The terminal node ZigBee module regularly detects the temperature data and sends it to the coordinator. The software flow of the terminal node is shown in Fig. 6.

Running result and analysis. In the laboratory environment, we use CC2430 and DS18B20 terminal wireless sensor node to measure the water temperature. The simple water temperature monitoring system is composed of two sets of wireless sensor node terminal and a CC2430 module coordinator. To develop the temperature monitoring system, we coordinate the coordinator program into a ZigBee node, connect the computer and coordinator with RS-232 line, and display the output content through serial debugging assistant. The serial port configuration is set to the following: 57600bps, 8-bit data bits, one stop bit, and an unremarkable parity bit. The coordinator starts to look for the available network available channels for networking. The sensor terminal node program is connected to a ZigBee module as the terminal node, and the terminal node is connected to the coordinator. The terminal node detects the temperature at regular intervals and then transfers temperature information to the coordinator.

\section{Conclusion}

This paper proposes a WSN scheme based on ZigBee technology to realize temperature monitoring in a certain area. The CC2430 module and the temperature sensor DS18B20 are designed as the terminal node of the wireless temperature sensor network. By using a star network structure, the terminal node is connected with the coordinator to establish a complete wireless temperature sensor network. The coordinator is a CC2430 module, which connects the coordinator through the serial port to the computer and makes the computer a human-computer interaction terminal. ZigBee protocol is adopted to improve the transmission efficiency of the wireless temperature sensor network, and to reduce the energy consumption of data transmission, which is suitable for the development of unmanned monitoring systems.

\section{Acknowledgements}

This study was supported by National Science Foundation of China (NSFC) under Grant No. 5153494 and The Excellent Dissertation Cultivation Funds of Wuhan University of Technology under Grant No. 2016-YS-044.

\section{References}

[1] Y.C. Ma: Research of Monitoring and Alarm System in Maritime Engine Room. Master Thesis of Dalian Maritime University (2009).

[2] X. Zhang, D.Y. Li, J.B. Sun and B. Chen: Monitoring and Alarming System of Marine Engine Room. Computer Engineering and Applications, Vol. 41 (2005), p. 229-232.

[3] P. Li, J. Li, L. Nie and B. Wang: Mishing, Research and Application of ZigBee Protocol Stack. International Conference on Measuring Technology and Mechatronics Automation, Vol.2 (2010), p.1031-1034.

[4] A. Wheeler: Commercial Applications of Wireless Sensor Networks Using ZigBee. IEEE Communications Magazine, Vol. 45 (2007), p.70-77.

[5] W.J. Qu: Design and Implementation of Granary Monitor System of Temperature and Humidity based on ZigBee Technology. Master Thesis of China Jiliang University (2014).

[6] R. Zhang, X. Zou, X. Yang and C. Guo: Development of ZigBee Wireless Temperature Monitoring Software System. Communications \& Network, Vol. 05 (2013), p.21-24. 